\title{
Testing the chiral behavior of the hadron spectrum*
}

\author{
Tanmoy Bhattacharya, ${ }^{a}$ Rajan Gupta, and Stephen Sharpe ${ }^{a}$ \\ aT-8 Group, MS B285, Los Alamos National Laboratory, Los Alamos, New Mexico 87545 U. S. A. \\ aPhysics Department, University of Washington, Seattle, WA 98195 U. S. A.
}

We analyze the chiral behavior of the hadron spectrum obtained with quenched Wilson fermions on $17032^{3} \times 64$ lattices at $\beta=6.0$. We calculate masses of hadrons composed of both degenerate and non-degenerate quarks. We reduce the statistical errors in mass splittings by directly fitting to the ratio of correlation functions. We find significant deviations from a linear dependence on the quark mass, deviations that are consistent with the higher order terms predicted by quenched chiral perturbation theory. Including these corrections yields splittings in the baryon octet that agree with those observed experimentally. Smaller higher order terms are also present in $m_{\rho}$ and $m_{N}$. By contrast, the decuplet baryons are well described by a linear mass term. We find the decuplet splittings to be $30 \%$ smaller than experiment. We extrapolate our data to $a \rightarrow 0$ by combining with the GF11 results, and the best fit suggests that the quenched approximation is only good to $10-15 \%$.

\section{TECHNICAL DETAILS}

We calculate quark propagators using the simple Wilson action and both smeared Wuppertal and Wall sources. From these we construct three types of hadron correlators: wall source and point sink (WL), Wuppertal source and point sink (SL), and Wuppertal source and sink (SS). We use five values of quark mass given by $\kappa=0.135(C)$, $0.153(S), 0.155\left(U_{1}\right), 0.1558\left(U_{2}\right)$, and 0.1563 $\left(U_{3}\right)$, corresponding to pseudoscalar mesons of mass $2835,983,690,545$ and $431 \mathrm{MeV}$ respectively where we have used $1 / a=2.33 \mathrm{GeV}$ for the lattice scale. We use the three light quarks to extrapolate the data to the physical isospin symmetric light quark mass $\bar{m}=\left(m_{u}+m_{d}\right) / 2$. The physical value of strange quark lies between $S$ and $U_{1}$ and we use these two points to interpolate to it, and use $C$ for the charm mass.

Our overriding fitting criterion is to include as many time-slices as possible. We have not succeeded in developing an automated procedure that meets this objective and works in all cases when using two mass fits or incorporating the full covariance matrix. For the sake of unifor-

\footnotetext{
* Poster presented by Tanmoy Bhattacharya and Rajan Gupta. These calculations have been done on the CM5 at LANL as part of the DOE HPCC Grand Challenge program, and at NCSA under a Metacenter allocation.
}

Figure 1. Comparison of $m_{\mathrm{eff}}(t)$ for $U_{1} U_{1} U_{1} \mathrm{nu}-$ cleon correlators with SL and WL sources.

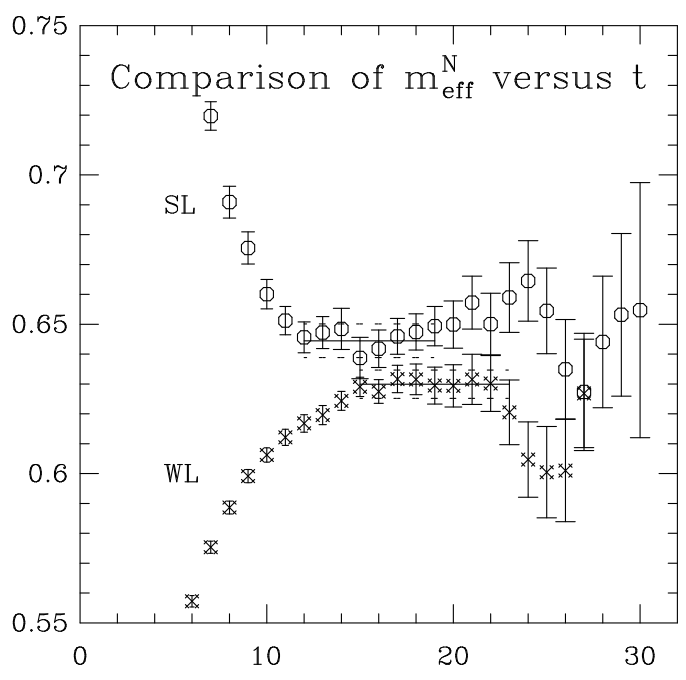

mity, therefore, we use results from single mass fits keeping only diagonal elements of the correlation matrix. In the cases we have checked, this does not significantly effect either the results or error estimates. All errors are obtained using single elimination Jackknife.

We find that masses extracted from WL correlators are systematically lower than those from SL or SS correlators. For the pion and rho chan- 
nels, this difference is $\sim 1 \sigma$, while for the nucleons it is $\sim 2-3 \sigma$ as exemplified in Fig. 11. This difference arises because the signal dies out before contamination from excited state is fully removed. In cases where the signal persists to large enough times, the estimates from the wall and Wuppertal sources converge to a value roughly in the middle. Since $S L$ and $S S$ results are highly correlated, we take as our best estimate the average given by $(2 \times W L+S L+S S) / 4$.

To extrapolate the hadron masses towards the chiral limit, and to test the forms predicted by quenched chiral perturbation theory (CPT), we make fits to both $1 / 2 \kappa$ and the non-perturbative definition of lattice quark mass described in Ref. [1]. The two give virtually identical results.

\section{MESON SPECTRUM}

A linear fit to $M_{\pi}^{2}$ using the six lightest $\left\{U_{i} U_{j}\right\}$ points gives $M_{\pi}^{2}=0.0013(5)+2.296(11) m_{q}$. With our current data we cannot determine whether the non-zero (though tiny) intercept is due to finite size effects, quenched chiral logs or the absence of chiral symmetry with Wilson fermions. The linear fit to $M_{\rho}\left(0.3296(59)+2.54(14) m_{q}\right)$ is shown in 2. The data show clear curvature, so we fit adding two types of corrections: $m^{3 / 2}$ (nonanalytic term due to chiral loops) and $m^{2}$. Both succeed in fitting the $\left\{U_{i} U_{j}\right\}$ and $\left\{S S, S U_{j}\right\}$ points. These fits give

$$
\begin{aligned}
& \kappa_{c} \quad=0.157131(9), \\
& \kappa_{\bar{m}} \quad=0.157046(9), \\
& a^{-1}\left(M_{\rho}\right)=2.330(41) \mathrm{GeV} \quad(m \mathrm{fit}), \\
& a^{-1}\left(M_{\rho}\right)=2.365(48) \mathrm{GeV} \quad\left(m^{3 / 2} \mathrm{fit}\right), \\
& a^{-1}\left(M_{\rho}\right)=2.344(42) \mathrm{GeV} \quad\left(m^{2} \text { fit }\right),
\end{aligned}
$$

Since the three fits to $M_{\rho}$ have comparable $\chi^{2}$, we cannot study CPT in detail.

Using linear fits for pseudoscalar and vector states we determine the lattice strange quark mass by first extrapolating $M_{K}^{2} / M_{\pi}^{2}, M_{K^{*}} / M_{\rho}$, or $M_{\phi} / M_{\rho}$ to $\bar{m}$, and then linearly interpolating between $U_{1}$ and $S$ until these quantities match their physical values. The results for $m_{s}(\overline{M S}$ scheme, run down to $2 \mathrm{GeV}$ ) are $m_{s}\left(M_{K}\right)=89(2) \mathrm{MeV}$
Figure 2. A linear fit to lightest $\operatorname{six}\left\{U_{i} U_{j}\right\}$ points.

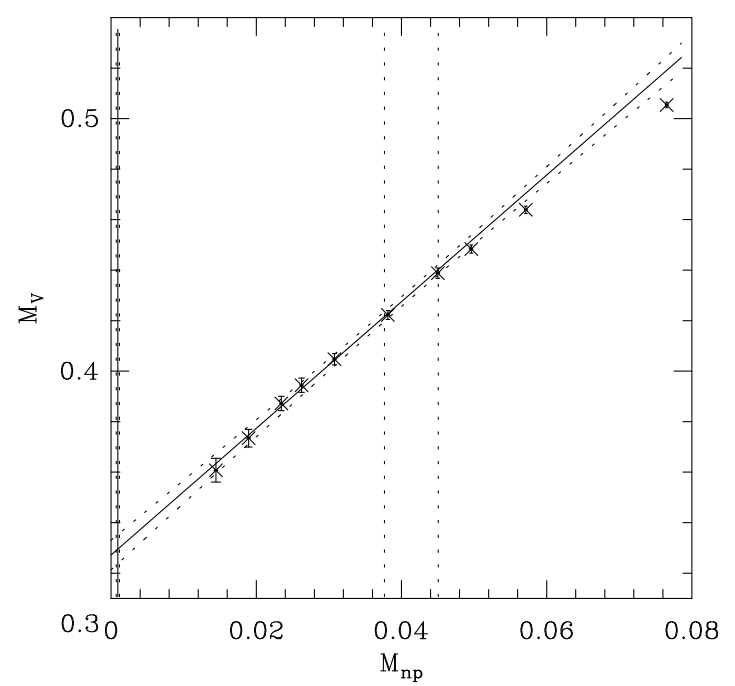

versus $m_{s}\left(M_{\phi}\right)=106(6) \mathrm{MeV}$, i.e. a $20 \%$ difference. Using $M_{K}^{2} / M_{\pi}^{2}$ to fix $m_{s}$ implies that $m_{s} \equiv 25 \bar{m}$ as we use the lowest order chiral expansion to fit the data. On the other hand, using $M_{\phi} / M_{\rho}$ gives $m_{s} / \bar{m} \approx 30$, in surprisingly good agreement with the next-to-leading chiral result [3]. The differences presumably result from a combination of quenching and discretization errors. We use $m_{\mathrm{s}}\left(M_{\phi}\right)$ here.

\section{BARYON SPECTRUM}

We calculate three types of correlation functions made up of flavors $A, B, C$. The spin- $1 / 2$ $\Sigma$ type (which also includes $\Xi$ and $N$ ) are labeled $A\{B C\}$ (symmetric in $B, C$ ); the $\Lambda$-like are $A[B C]$ (anti-symmetric in $B, C$ ); and the spin$3 / 2$ decuplet are $\{A B C\}$. With 4 flavors $S, U_{i}$, one can write 40 correlators of types $A\{B C\}$ and $A[B C]$ each. For $B \neq C$, $\mathrm{SU}(2)$ symmetry is broken and there is mixing between the $\Lambda$ and $\Sigma$ states. However, as explained in [1], for $\delta m t<<1$ (as is true for our data) the mixing can be ignored.

To study mass splittings we make fits to appropriate ratios of correlators for a given source and sink. For example, fits to $\Gamma_{\Sigma}(t) / \Gamma_{N}(t) \sim$ $e^{-\left(M_{\Sigma}-M_{N}\right) t}$, give $\left(M_{\Sigma}-M_{N}\right)$ directly. This has the advantage of both reducing some of the sources of systematic errors and of improving the 
Figure 3. Test for chiral corrections in $M_{\Sigma}-M_{\Lambda}$. The extrapolated value is at the extreme left.

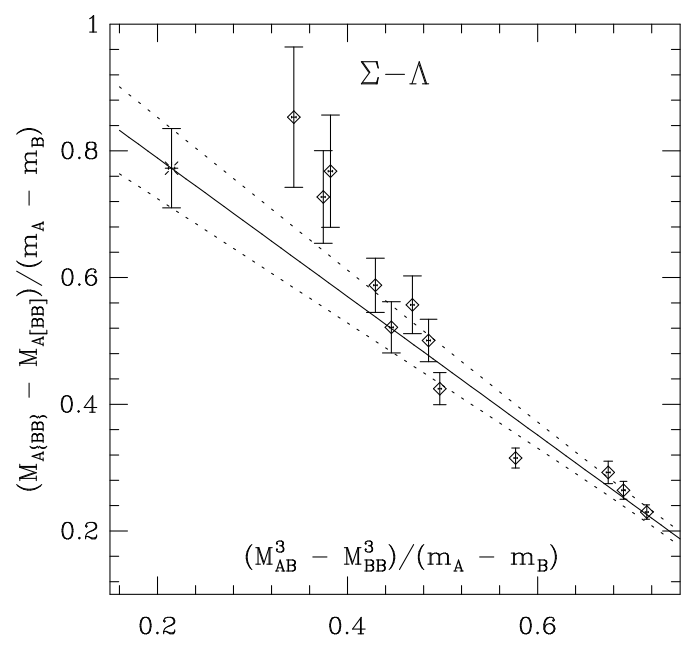

statistical errors (the errors in $\Delta M$ are $3-5$ times smaller than those obtained from individual fits).

Our fits are motivated by the results of quenched CPT worked out in Ref. \#t and are labeled chiral (non-analytic $m_{q}^{3 / 2}$ terms) and analytic $\left(m_{q}^{2}\right.$ terms). We first consider the octet hyperfine splitting, " $\Sigma-\Lambda$ ", given by

$$
\begin{gathered}
\frac{M_{A\{B B\}}-M_{A[B B]}}{m_{A}-m_{B}}=(-8 D / 3)+c_{1} \frac{M_{A B}^{3}-M_{B B}^{3}}{m_{A}-m_{B}} \\
+c_{1}^{\prime} \frac{M_{A A}^{3}-M_{B B}^{3}}{m_{A}-m_{B}}+d_{1}\left(m_{A}+m_{B}\right)+d_{1}^{\prime} m_{B},
\end{gathered}
$$

where $M_{A B}$ is the mass of the meson with flavor $\bar{A} B$, etc. The constants $c_{i}$ and $d_{i}$ can be expressed in terms of parameters of the quenched chiral Lagrangian. For reasonable choices of these parameters, one expects $\left|c_{1}^{\prime}\right| \ll\left|c_{1}\right|$. There is no useful information concerning $d_{1}$ or $d_{i}^{\prime}$.

We fit our results in two ways. First, we assume that the $c_{1}$ term is dominant, and make a linear fit (called chiral) to the $12 \mathrm{SU}(2)$ symmetric non-degenerate points as shown in Fig. 3 . The sizable non-zero slope shows that significant terms of higher order than linear in the quark mass are needed. In our second fit we test to see whether our data can be represented as well with analytic terms. We find, by trial and error, that

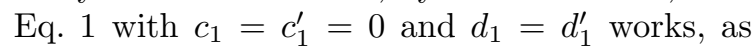
shown in Fig. 1. The data show significant curvature, so we have extrapolated to the physical
Figure 4. Quadratic fit including baryons composed of completely non-degenerate quarks.

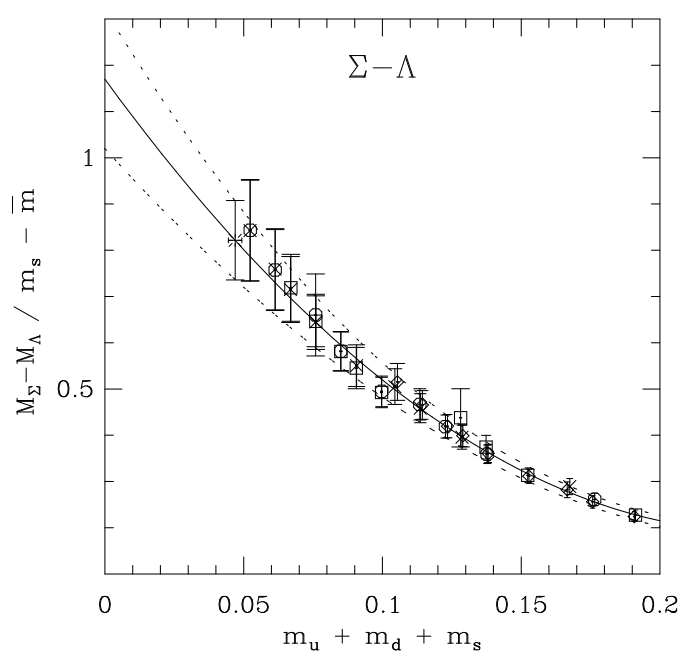

point using a quadratic fit, corresponding to $m_{q}^{3}$ terms in baryon masses! The extrapolated value of $M_{\Sigma}-M_{\Lambda}$ from the two fits are given in Table 1; these agree with each other and with the experimental splitting.

Next we consider the " $\Sigma-N$ " splitting,

$$
\begin{gathered}
\frac{M_{A\{C C\}}-M_{B\{C C\}}}{m_{A}-m_{B}}=2(F-D)+c_{2} \frac{M_{A C}^{3}-M_{B C}^{3}}{m_{A}-m_{B}} \\
+c_{2}^{\prime} \frac{M_{A A}^{3}-M_{B B}^{3}}{m_{A}-m_{B}}+d_{2}\left(m_{A}+m_{B}\right)+d_{2}^{\prime} m_{C} .
\end{gathered}
$$

CPT suggests that $\left|c_{2}^{\prime}\right|<\left|c_{2}\right|$. Thus we fit the data assuming $c_{2}$ is the dominant coefficient (chiral). Our best trial and error attempt with analytic corrections assumes $c_{2}=c_{2}^{\prime}=0$ and $d_{2}=d_{2}^{\prime} / 4$. Again there is definite evidence for curvature even though the collapse of data on to a single curve is not as good as in the $\Sigma-\Lambda$ case. The difference in estimates of $M_{\Sigma}-M_{N}$ given in Table 1 is indicative of this.

Thirdly, we consider the difference " $\Xi-N$ ":

$$
\begin{gathered}
\quad \frac{M_{A\{B B\}}-M_{A\{C C\}}}{m_{B}-m_{C}}=4 F+c_{3} \frac{M_{A B}^{3}-M_{A C}^{3}}{m_{B}-m_{C}} \\
+c_{3}^{\prime} \frac{M_{B B}^{3}-M_{C C}^{3}}{m_{B}-m_{C}}+d_{3}\left(m_{B}+m_{C}\right)+d_{3}^{\prime} m_{A} .
\end{gathered}
$$

Here there is no expectation that $c_{3}$ and $c_{3}^{\prime}$ should be substantially different in magnitude. Nevertheless, our "chiral" fit assumes $c_{3}$ is the dominant coefficient, and fits the data reasonably well. A quadratic fit to the average quark mass (i.e. 
Table 1. Octet mass splittings. Our most reliable values are from " $m{ }^{2}$ " fits.

\begin{tabular}{|c|r|r|r|}
\hline Fit & " $m^{3 / 2} "$ & " $m^{2} "$ & Expt. \\
\hline$M_{\Sigma}-M_{N}$ & $223(15)$ & $269(22)$ & 253 \\
\hline$M_{\Xi}-M_{N}$ & $339(20)$ & $358(21)$ & 375 \\
\hline$M_{\Sigma}-M_{\Lambda}$ & $78(14)$ & $84(9)$ & 77 \\
\hline
\end{tabular}

Figure 5. Linear fit to spin-3/2 baryon data. The four degenerate cases are shown with octagons.

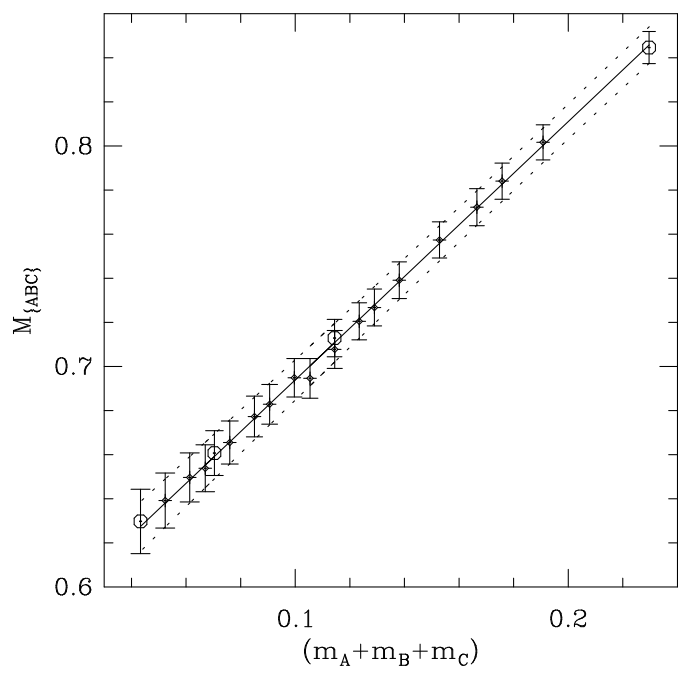

assuming $d_{3}=d_{3}^{\prime}$ ) is slightly better. Again, the extrapolated values in Table 1 are consistent. The important point is that in all three cases the higher order corrections are substantial.

Since keeping only terms linear in the quark masses provides a poor description of most mass differences, we include an $m_{q}^{3 / 2}$ (or $m^{2}$ ) term in our fits to the four degenerate combinations yielding $M_{N}=1070(35) \mathrm{MeV}(1072(31) \mathrm{MeV})$. In each case we have included the same form for the higher order corrections in $M_{\rho}$ when determining $a$ and $\bar{m}$. For our best estimate we take the mean, $M_{N}=1071(35) \mathrm{MeV}$.

In the spin-3/2 baryons we can form 20 states with our four masses, and none of these mix with each other. As shown in Fig. 5, the linear fit $M_{\Delta} a=0.581(13)+1.13(5)\left(m_{A}+m_{B}+m_{C}\right) a$ works for all 20 quark combinations $\mathrm{ABC}$. The mass differences calculated from this fit turn out to be $\approx 25 \%$ smaller than the experimental val- ues. Note that this discrepancy is worsened if we use $m_{s}\left(M_{K}\right)$. Linear extrapolation of $M_{\Delta}-M_{N}$ calculated from the ratios of correlators gives 318(30) MeV. Including an $m_{q}^{3 / 2}$ or $m_{q}^{2}$ term in both $M_{\Delta}-M_{N}$ and $M_{\rho}$ fits gives 365(44) and $347(39)$ respectively. These estimates are slightly higher than the experimental value $293 \mathrm{MeV}$.

\section{CONTINUUM RESULTS}

Our best estimates at $\beta=6.0$ with linear extrapolations in $m_{q}$ using only the $U_{i}$ quarks are
$M_{N} / M_{\rho}=1.412(35)$
Expt: 1.22 ,
$M_{\Delta} / M_{\rho}=1.800(47)$
Expt: 1.60 ,
$M_{\Delta} / M_{N}=1.275(36)$
Expt: 1.31 .

The GF11 collaboration [2 has claimed, based on data at $\beta=5.7,5.93$, and 6.17 , that these ratios have a significant slope when extrapolated to $a=0$. We update their "012" and "4" sink fits. The change, on adding our point, to the "012" fit is from $M_{N} / M_{\rho}=1.28(7)$ to 1.30(6) and from $M_{\Delta} / M_{\rho}=1.61(8)$ to $1.62(7)$. The $\chi^{2} /$ dof for the new fits are 2.1 and 0.85 respectively. The analogous numbers for the sink " 4 " data are $1.33(9) \rightarrow 1.38(7)$ and $1.68(10) \rightarrow 1.73(10)$ with $\chi^{2} /$ dof equal to 1.2 and 0.86 respectively.

The difference between the two fits is due to the "012" and "4" sink data at $\beta=5.7$. If we neglect the point at strongest coupling, $\beta=5.7$, then the remaining three points again show smaller $a$ dependence, similar to the sink " 4 " case. Thus, our preferred estimates are $M_{N} / M_{\rho}=1.38(7)$ and $M_{\Delta} / M_{\rho}=1.73(10)$ from sink "4" fit. The ambiguity in the extrapolation makes it clear that data at more values of $\beta$ are needed in order to reliably determine continuum results.

\section{REFERENCES}

1. T. Bhattacharya, R. Gupta, G. Kilcup, S. Sharpe, LA-UR-95-2354, hep-lat/9511???

2. F. Butler et al.(GF11 Collaboration), Nucl. Phys. B430 (1994) 179.

3. J. Donoghue et al., Phys. Rev. Lett. 69 (1992) 3444 .

4. J. Labrenz and S. Sharpe, in preparation. 